not involve passing on truths from producer to consumer, but takes the form of fallible judgements made in situations in which all the desired information is not available. This applies to scientific collaborations and governmental projects. "The speed of politics," they write in a characteristically succinct and clever remark, "exceeds the speed of scientific consensus formation." This leads them to defend their seemingly pedestrian conclusion that governments should rely on experts as "the best way to distil human experience of an uncertain world" - even when those experts have no final answers.

The authors needed to defend themselves. Some sociologists sharply attacked their 2002 article on the grounds that the technical and political phases of a controversy cannot be neatly separated, and that governments often use expert advice on technical questions ("Does activity X meet our standards for safety?") to disguise political questions ("Should we do activity X?"). This problem is best met, critics contended, by expanding public involvement in technological decision-making, breaking down the boundaries between experts and laypeople.

Rethinking Expertise argues the reverse, namely that disguised political questions would be combated more effectively by a more rigorous understanding of whose technical expertise really should weigh in on technological decision-making. This is what Collins and Evans call the "problem of extension", or "who is entitled to contribute to the technical component of a technological decision". If an extension is too technocratic, it will foster distrust; if it is too broad, there is a risk of invoking 'technological populism, which means decisions could be overly influenced by politics or lifestyle choice.

The authors address the problem of extension by offering a "periodic table of expertise" - an attempt "to classify all the types of expertise that might be brought to bear on a technological problem". These include about a dozen varieties, ranging from language-speaking skills to higher, domain-restricted categories. There is 'contributory' expertise from active practitioners of a field (the usual concept of an expert) and 'interactional' expertise, which involves fluency in a field in the absence of contribution. Sociologists and journalists have interactional expertise, and it is widespread in science (in which scientists must interact across disciplines), in management and in peer-reviewing.

Interactional expertise is one of the authors' most novel ideas. They dramatize it by reporting the results of several Turing-test-like imitation games. In one, judges could not distinguish between colour perceivers and colour-blind participants, probably because the latter had been immersed since birth in the language of colour and acquired the ability to pass as perceivers. In another, judges could not pick out Collins in a group of bona fide gravitationalwave researchers.

These results have startling implications. For instance, Evans has shown that minorities can acquire enough interactional expertise of mainstream culture to foil attempts to pick them out by questioning alone. This suggests that if minorities live by different values, it is not through ignorance but choice.

Collins and Evans put their points vividly, with elegant language and diagrams. They admit that there is more to technological decision-making than "sorting out the appropriate groups of experts" and that they are only addressing the technical phase of public controversies. They modestly claim to be only setting "the ball rolling".

Their book starts to lay the groundwork for solving a critical problem - how to restore the force of technical scientific information in public controversies, without importing disguised political agendas.

Robert P. Crease is a professor in and chairman of the Department of Philosophy, Stony Brook University, New York 11794, USA.

\title{
Blurring our edges
}

\section{Enhancing Evolution: The Ethical Case for Making Better People \\ by John Harris \\ Princeton University Press: 2007. 260 pp. \\ $\$ 27.95, £ 16.95$ \\ Ending Aging: The Rejuvenation \\ Breakthroughs That Could Reverse \\ Human Aging in Our Lifetime \\ by Aubrey de Grey and Michael Rae \\ St. Martin's Press: 2007.400 pp. $\$ 26.95$}

\section{Judy Illes}

Two new books explore the potential for improving the human condition, one from a medical-ethics viewpoint and the other from a more hands-on biogerontological perspective. Each identifies physical health and cognition as the key areas for attention in the short term, and each speculates on future possibilities for life extension - going well beyond what might be imaginable, or ethical, today.

John Harris's book Enhancing Evolution discusses the future of humankind and of research, and looks at issues such as reproductive choice, designer children and disability. Harris systematically analyses historical and contemporary writings to support his central claim that the pursuit of methods to enhance the human condition is a moral obligation. His treatment of opinions by conservative thinkers and phi-

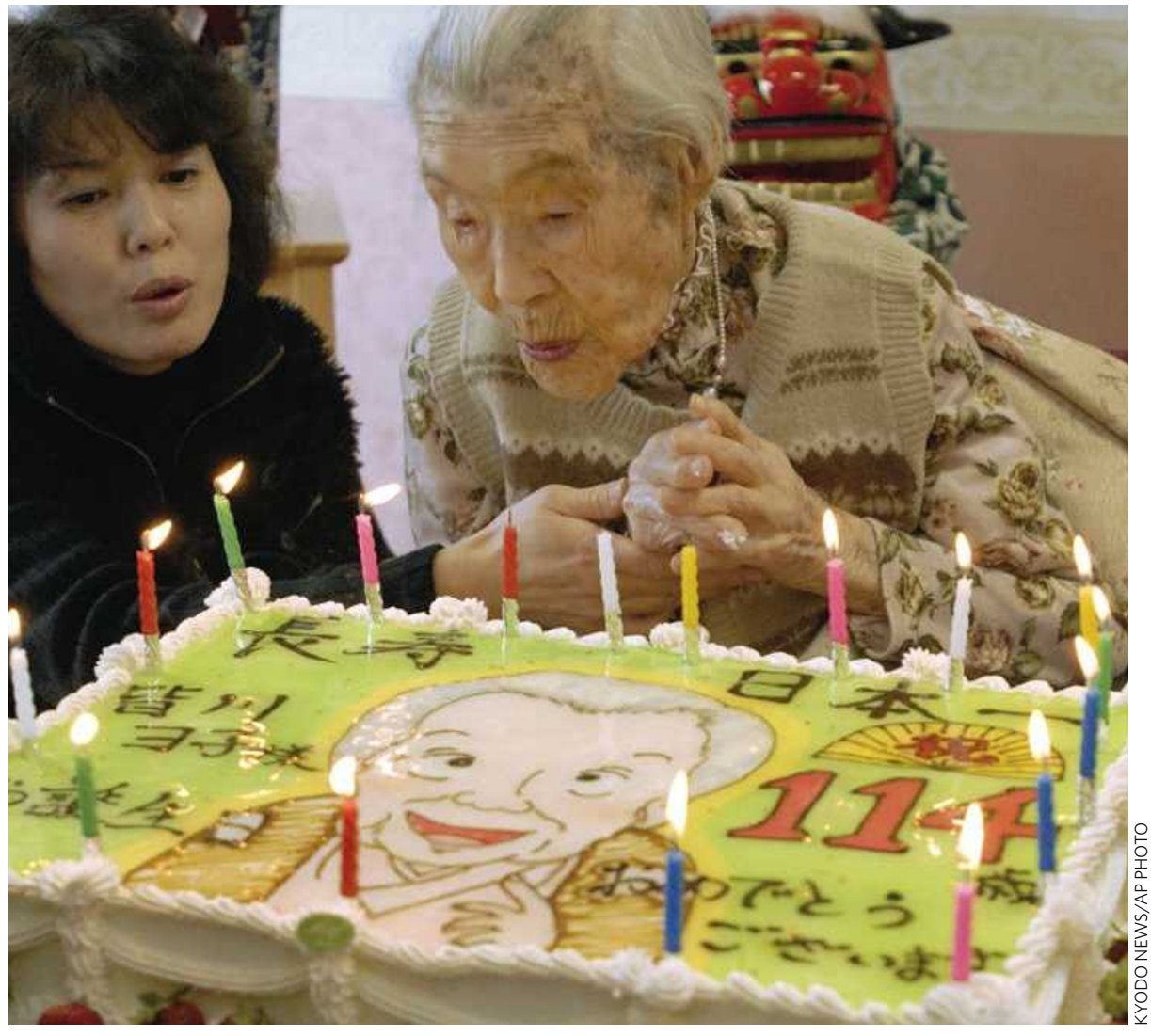

Could we all live longer than Japan's Yone Minagawa, who reached her 114th birthday? 
losophers such as Leon Kass, Jürgen Habermas and Francis Fukuyama is at times abrasive. But, whether or not one agrees with Harris's position, he dissects their opposing claims with impressive effectiveness. He makes a persuasive case that today's biotechnologies - including neuroengineering, stem-cell research and cloning to improve bodies and brains - are on the continuum of an age-long pursuit by humans to improve themselves and therefore, he argues, are permissible and morally essential.

I question the author's contention that choices such as non-medical sex selection can be as straightforward as picking a white shirt over a Hawaiian one, or that "inevitability is the only reason dying of old age is acceptable". But I do agree that everyone - scientists, ethicists and the public — has a role in the research enterprise, not least because of benefit sharing. That is not to say that we should all be drafted into research, and certainly no end would justify exploitation of children and people with diminished abilities, but service to society by its members is an honourable goal.

Aubrey de Grey and Michael Rae's Ending Aging is a more 'new-wave' treatment of enhancement, longevity and immortality. Through chapters with catchy titles, such as 'Putting Zombies to Rest', and gripping language - "I shall look forward to shaking your hand in a future where engineered senescence is a reality" - the authors call for research that will deliver a life expectancy to match that of Methuselah, the oldest of the people whose age (969 years) is mentioned in The Bible.

Ending Aging guides the reader through a maze of advances in molecular and cellular biology that could lead to anti-ageing therapies, which the authors term SENS (for 'strategies for negligible senescence'). The targets are a range of cancer-causing nuclear and mitochondrial mutations, intracellular and extracellular debris, and molecular crosslinks that contribute to pathological decay over a lifetime.

The authors rather unnecessarily brand ageing as repugnant and a curse, and use their book to preach on fund-raising opportunities. By contrast, de Grey's 2005 publication on 'Life extension, human rights, and the rational refinement of repugnance' in the Journal of Medical Ethics (co-edited by Harris) is still an edgy but more dignified treatise.

The freedom to pursue ways to enhance human mental and physical capacities and to eliminate negative aspects of the human condition, such as suffering and death, is a fundamental tenet of the trans-humanist movement. Although seemingly worthy, there are problems ahead for the futurists, including for Harris, de Grey and Rae. At what age should life-prolonging measures begin - in utero, when development of the nervous system peaks in the mid-20s, or much later? What would become of natural rhythms in wisdom and folk psychology, and how will the roles of elders be redefined? Would we immortalize some and punish others by withholding life-prolonging intervention - reconfiguring the term 'life sentence' and introducing new systems of justice for the parallel mortal and immortal populations?

Will people be able to step off the immortality train when they are ready? Will suicide increase if we move life expectancy into the hundreds? We cannot remove longevity like a pair of spectacles. And abuse is reported by $1-10 \%$ of old people surveyed, mostly directed against women. If society shows increasingly scant regard for centenarians, how will it treat people of 1,000 years old?

Let's not throw away today for tomorrow. Ending Aging is likely to appeal to those already converted to the authors' views, and perhaps will find some traction among those who are more curious than interested in deeper scientific engagement. I end unconvinced by Harris's view, but my interest was piqued by the diverse ways in which physical and mental health, autonomy, culture, and respectful and caring community systems can be realized. After we have maximized human well-being and compressed morbidity to minimize suffering (see A. Kalache et al. Bull. World Health Organ. 80, 243-244; 2002), then let's think about life extension, step-by-step, one decade at a time.

Judy Illes is professor of neurology at the National Core of Neuroethics, University of British Columbia, Vancouver V6T 2B5, Canada.

\section{Archimedes' secrets revealed}

\section{The Archimedes Codex \\ by Reviel Netz \& William Noel \\ Weidenfeld \& Nicolson/Phoenix/ \\ Da Capo Press: 2007. 336/336/320 pp. \\ €18.99(hbk)/£8.99/\$27.50 (pbk)}

\section{Brian Clegg}

In April 2004, biologist Lewis Wolpert won a debate at the Royal Institution in London entitled 'Who was the first scientist?' He championed Archimedes. Although I reckon Roger Bacon comes closer to deserving that label, the result reflects the respect still felt for Archimedes' work. Yet our knowledge of his output has never been complete. The Archimedes Codex describes the recent uncovering of text from the Archimedes canon not read since the parchment forming the codex was reused to make a prayer book in 1229 .

Only three of Archimedes' books in the original Greek are known, two of which have been lost since first being transcribed. The third, this codex, had never been fully deciphered. Access to the earliest known version (from the tenth century) was important because of a recent shift in interpretation of Greek texts. The Greeks approached mathematics visually. The diagrams and the exact wording used are crucial in understanding the thinking of a Greek mathematician - yet when the lost Archimedes manuscripts were transcribed, no thought was given to capturing the visual nature of the information.

In The Archimedes Codex, Reviel Netz and William Noel contribute alternate chapters, with Noel describing the history of the codex and the work undertaken to decipher it, and Netz exploring Archimedes' mathematics. This discontinuous style is initially unnerving - both authors write in the first person, and it isn't clear that the 'I' of chapter 1 is not the same person as the 'I' in chapter 2. But it soon settles into an engaging account.

Noel, director of the deciphering project, explains the intricacies of dealing with a manu-

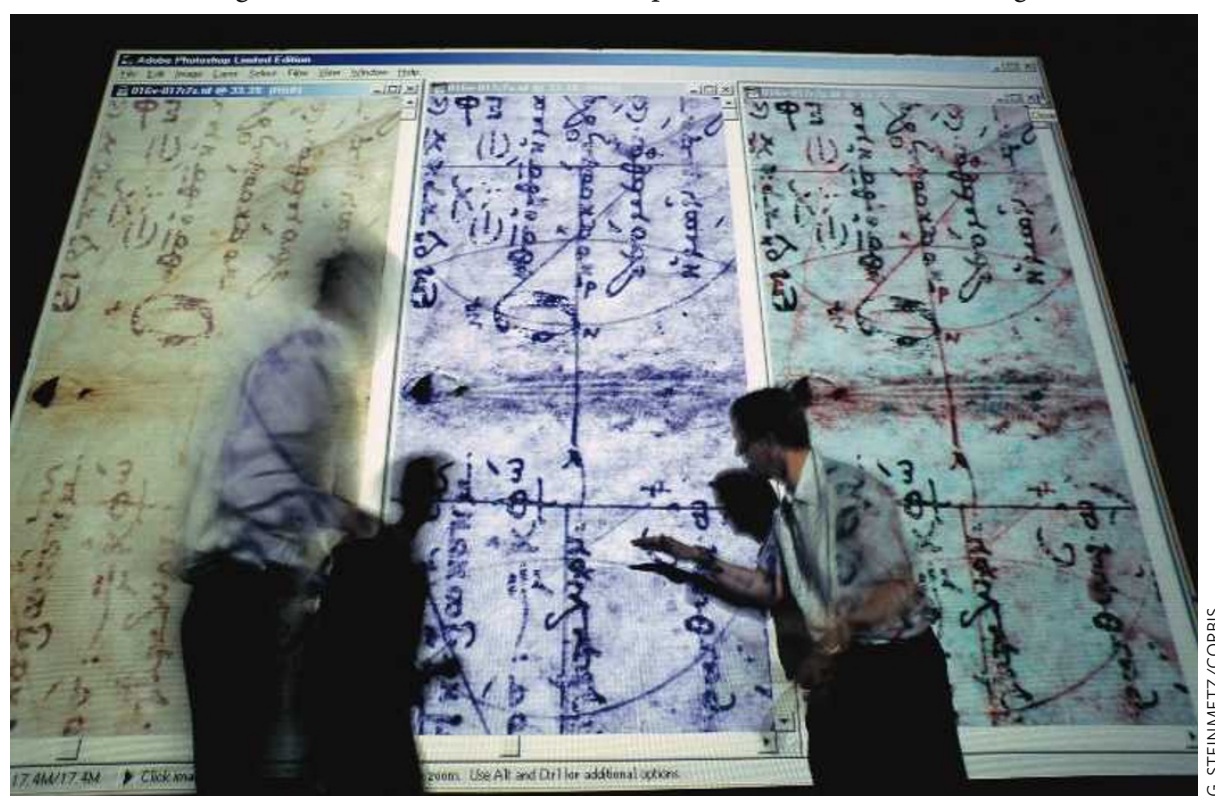

Analysing the Archimedes palimpsest with different wavelengths of light. 